Pacific

Journal of

Mathematics

\title{
QUASISYMMETRIC HOMEOMORPHISMS ON REDUCIBLE CARNOT GROUPS
}

Xiangdong Xie

Volume $265 \quad$ No. 1

September 2013 


\title{
QUASISYMMETRIC HOMEOMORPHISMS ON REDUCIBLE CARNOT GROUPS
}

\author{
XiAngdong XIE
}

\begin{abstract}
We show that quasisymmetric homeomorphisms between (most) reducible Carnot groups are bilipschitz. This implies rigidity for quasi-isometries between certain negatively curved homogeneous manifolds. The proof uses Pansu's differentiability theorem for quasisymmetric homeomorphisms between Carnot groups.
\end{abstract}

\section{Introduction}

We study quasisymmetric homeomorphisms between reducible Carnot groups. The main result says that in most cases, the quasisymmetric homeomorphism must be bilipschitz.

A Carnot group is reducible if it is isomorphic to the direct product of two Carnot groups. Otherwise, a Carnot group is called irreducible. A reducible Carnot group $G$ can be written as $G=G_{0} \times G_{1} \times \cdots \times G_{m}$, where $G_{0}$ is abelian (i.e., isomorphic to some $\left.\mathbb{R}^{n}\right)$, and $G_{j}(1 \leq j \leq m)$ is nonabelian irreducible. Such a decomposition is not unique in general; see Example 2.1.

All Carnot groups in this paper are equipped with the Carnot metric (see Section 3).

Theorem 1.1. Let $F: G \rightarrow G^{\prime}$ be a quasisymmetric map between two Carnot groups. Suppose $G$ is reducible and admits a direct product decomposition of irreducible Carnot groups where at least two of the factors are not isomorphic. Then $F$ is bilipschitz.

The same claim remains open in the case when $G$ is isomorphic to a direct product $N \times \cdots \times N$, where $N$ is nonabelian irreducible.

Quasisymmetric homeomorphisms between general metric spaces are quasiconformal. In the case of Carnot groups (and of Loewner spaces more generally), a map is quasisymmetric if and only if it is quasiconformal (see [Heinonen and Koskela 1998]).

Partially supported by NSF grant DMS-1265735.

MSC2010: 53C17, 53C23, 30L10.

Keywords: quasisymmetric homeomorphism, Carnot groups, Pansu differentiability. 
Theorem 1.1 has consequences for the rigidity of quasi-isometries between certain negatively curved homogeneous manifolds. Recall that a quasi-isometry between two metric spaces is an almost isometry if it preserves distance up to an additive constant. A quasi-isometry between two negatively curved spaces induces a quasisymmetric homeomorphism between the ideal boundaries (of the negatively curved spaces), where the ideal boundaries are equipped with visual metrics. Conversely, under mild conditions on the negatively curved spaces, each quasisymmetric homeomorphism between the ideal boundaries is the boundary map of a quasi-isometry; see [Bonk and Schramm 2000]. Similarly, almost isometries between negatively curved spaces correspond to bilipschitz maps between the ideal boundaries [ibid.]. On the other hand, Carnot groups arise as the ideal boundary of certain negatively curved homogeneous manifolds (see below for more details). Hence a direct consequence of Theorem 1.1 is that each quasi-isometry between certain negatively curved homogeneous manifolds is an almost isometry.

Heintze [1974] characterized homogeneous manifolds with negative sectional curvature (HMNs): Each HMN is isometric to a simply connected solvable Lie group $S$ equipped with a left invariant Riemannian metric, and furthermore $S=N \rtimes \mathbb{R}$ is a semidirect product of a nilpotent Lie group $N$ with $\mathbb{R}$, where $\mathbb{R}$ acts on $N$ by expanding (and contracting) automorphisms; conversely, every semidirect product as above admits a left invariant Riemannian metric with negative sectional curvature (hence is an HMN). The ideal boundary of an HMN $S=N \rtimes \mathbb{R}$ can be naturally identified with (the one-point compactification of) $N$. On the other hand, each Carnot group $N$ is a simply connected nilpotent Lie group having a one-parameter family of dilations (see Section 3 for more details). These dilations induce an action of $\mathbb{R}$ on the Carnot group by expanding (and contracting) automorphisms, so there is an HMN $N \rtimes \mathbb{R}$ associated with each Carnot group $N$. It follows that each Carnot group can be identified with the ideal boundary of some HMN. Hence Theorem 1.1 implies that each quasi-isometry between these HMNs is an almost isometry.

We next make some comments about the proof of Theorem 1.1. A main step in the proof is to show that the quasisymmetric homeomorphism preserves a certain foliation. Then the arguments in [Shanmugalingam and Xie 2012] show that the quasisymmetric homeomorphism is bilipschitz. To show that the quasisymmetric homeomorphism preserves a foliation, one first proves that infinitesimally it preserves a foliation. The global result then follows by integration. Recall that Pansu's differentiability theorem (see [Pansu 1989] or Section 3) says that a quasisymmetric homeomorphism $F: G \rightarrow G^{\prime}$ between Carnot groups is Pansu-differentiable a.e., and the Pansu differential is a.e. a graded isomorphism between the two Carnot groups. Under the assumption of Theorem 1.1, we show that there are (proper) connected and simply connected subgroups $N \subset G$ and $N^{\prime} \subset G^{\prime}$ such that $\phi(N)=N^{\prime}$ for every graded isomorphism $\phi: G \rightarrow G^{\prime}$; see Section 2 . 
In Section 2 we show that graded isomorphisms between reducible Carnot algebras preserve certain subalgebras, which implies that graded isomorphisms between reducible Carnot groups preserve certain subgroups (as indicated in the preceding paragraph). And in Section 3 we show that quasisymmetric homeomorphisms are bilipschitz.

\section{Graded isomorphisms of Carnot algebras}

In this section we show that graded isomorphisms between reducible Carnot algebras preserve certain subalgebras. This implies that graded isomorphisms between reducible Carnot groups preserve certain subgroups (see Section 3).

A Carnot Lie algebra is a finite-dimensional Lie algebra $\mathscr{G}$ together with a direct sum decomposition $\varphi_{G}=V_{1} \oplus V_{2} \oplus \cdots \oplus V_{r}$ of vector subspaces such that $\left[V_{1}, V_{i}\right]=V_{i+1}$ for all $1 \leq i \leq r$, where we set $V_{r+1}=\{0\}$. The integer $r$ is called the degree of nilpotency of $\mathscr{G}$. Every Carnot algebra $\mathscr{G}=V_{1} \oplus V_{2} \oplus \cdots \oplus V_{r}$ admits a one-parameter family of automorphisms $\lambda_{t}: \varphi \rightarrow \varphi$ for $t \in(0, \infty)$, where $\lambda_{t}(x)=t^{i} x$ for $x \in V_{i}$. Let $\varphi=V_{1} \oplus V_{2} \oplus \cdots \oplus V_{r}$ and $\varphi^{\prime}=V_{1}^{\prime} \oplus V_{2}^{\prime} \oplus \cdots \oplus V_{s}^{\prime}$ be two Carnot algebras. A Lie algebra homomorphism $\phi: \mathscr{G} \rightarrow \mathscr{G}^{\prime}$ is graded if $\phi$ commutes with $\lambda_{t}$ for all $t>0$; that is, if $\phi \circ \lambda_{t}=\lambda_{t} \circ \phi$. We observe that $\phi\left(V_{i}\right) \subset V_{i}^{\prime}$ for all $1 \leq i \leq r$.

A Carnot algebra $G$ is called reducible if there exist two nontrivial Carnot algebras $\varphi_{1}$ and $\mathscr{G}_{2}$ and a graded isomorphism between $\varphi_{\text {and }} \varphi_{1} \oplus \mathscr{G}_{2}$. It is called irreducible otherwise. The finite dimensionality implies that every reducible Carnot algebra $\varphi$ can be written as a direct sum of Carnot algebras $\mathscr{G}_{=} \mathscr{G}_{0} \oplus \mathscr{G}_{1} \oplus \cdots \oplus \mathscr{G}_{m}$, where $\mathscr{G}_{0}$ is abelian, and each $\mathscr{G}_{i}$ with $i \geq 1$ is nonabelian and irreducible.

Let $\mathscr{G}=V_{1} \oplus V_{2} \oplus \cdots \oplus V_{r}$ be a Carnot algebra. When $\mathscr{G}$ is reducible, it also has a decomposition $\mathscr{G}=\mathscr{G}_{0} \oplus \mathscr{G}_{1} \oplus \cdots \oplus \mathscr{G}_{m}$ as a direct sum of an abelian factor and irreducible nonabelian factors. We are interested in the question whether graded isomorphisms preserve such decompositions (after possibly permuting the factors). This question is equivalent to the uniqueness problem of such a decomposition. In general the decomposition is not unique, as the following example shows. The author thanks Bruce Kleiner for suggesting the example.

Example 2.1 (Kleiner). Let $\mathscr{G}$ be a nonabelian irreducible Carnot algebra, and let $f: \varphi \rightarrow \mathbb{R}^{m}$ be a nontrivial Lie algebra homomorphism into an abelian group. Let $G(f) \subset \mathscr{G} \oplus \mathbb{R}^{m}$ be the graph of $f$. Then $G(f)$ is a Carnot algebra (being isomorphic to $\mathscr{G})$, and $\mathscr{G} \oplus \mathbb{R}^{m}$ has two different decompositions $\mathscr{G} \oplus \mathbb{R}^{m}=G(f) \oplus \mathbb{R}^{m}$. Alternatively, let $g: \mathscr{G} \oplus \mathbb{R}^{m} \rightarrow \mathscr{G} \oplus \mathbb{R}^{m}$ be the map given by $g(x, a)=(x, a+f(x))$. Then $g$ is a graded isomorphism and it does not preserve the factor $\mathscr{G}$.

Despite this example, we show that graded isomorphisms always preserve the abelian factor (Proposition 2.4) and, in the case of a trivial abelian factor, preserve 
the decomposition after possibly permuting the factors (Proposition 2.5).

Definition 2-1. Let $\mathscr{G}$ be a Lie algebra and $x \in \mathscr{G}$. Define $d(x)=\operatorname{dim}(\operatorname{ker}(\operatorname{ad} x))$, where ad $x: \mathscr{G} \rightarrow \mathscr{G}$ is the linear map given by ad $x(y)=[x, y]$.

Lemma 2.2. If $d(x)=\operatorname{dim} \mathscr{G}>1$ for some $x \in V_{1} \backslash\{0\}$, then $\mathscr{G}$ is reducible.

Proof. Note $[x, y]=0$ for all $y \in \mathscr{G}$. Let $\mathscr{G}_{1}$ be the one-dimensional subspace of $V_{1}$ spanned by $x$, and let $W$ be a complementary subspace of $\mathscr{G}_{1}$ in $V_{1}$. Set $\mathscr{G}_{2}=W \oplus V_{2} \oplus \cdots \oplus V_{r}$. Then $\mathscr{G}_{=}=\mathscr{G}_{1} \oplus \mathscr{G}_{2}$ is a direct sum of vector subspaces. The assumption on $x$ now implies that $\mathscr{G}_{1}$ and $\mathscr{G}_{2}$ are ideals of $\varphi_{\text {and }}$ that both $\mathscr{G}_{1}$ and $\mathscr{G}_{2}$ are Carnot algebras. Hence $\mathscr{G}$ is reducible.

The next lemma provides an intrinsic characterization of the abelian factor $\mathscr{G}_{0}$.

Lemma 2.3. Let $\varphi=V_{1} \oplus V_{2} \oplus \cdots \oplus V_{r}$ be a Carnot algebra, and consider a direct sum decomposition $\mathscr{G}_{=} \mathscr{G}_{0} \oplus \mathscr{G}_{1} \oplus \cdots \oplus \mathscr{G}_{m}$ of $\mathscr{G}_{\text {into }}$ an abelian factor and irreducible nonabelian factors. Let $x \in V_{1}$. Then $x \in \mathscr{G}_{0}$ if and only if $d(x)=\operatorname{dim} \mathscr{G}$. Proof. It is clear that $x \in \mathscr{G}_{0}$ implies $d(x)=\operatorname{dim} \mathscr{G}$. We assume $d(x)=\operatorname{dim} \mathscr{G}$ and shall prove that $x \in \mathscr{G}_{0}$. Note $[x, y]=0$ for all $y \in \mathscr{G}$. Write $x=x_{0}+x_{1}+\cdots+x_{m}$ with $x_{i} \in \mathscr{G}_{i} \cap V_{1}$. Suppose $x \notin \mathscr{G}_{0}$. Then $x_{i} \neq 0$ for some $i \geq 1$. Since $\left[x_{i}, y\right]=[x, y]=0$ for all $y \in \mathscr{G}_{i}$, Lemma 2.2 implies $\mathscr{G}_{i}$ is reducible, contradicting the assumption.

Recall that the goal of this section is to show that a graded isomorphism of reducible Carnot algebras preserves certain Lie subalgebras. The case when the abelian factor is nontrivial is covered by Proposition 2.4.

Proposition 2.4. Let $\mathscr{G}_{=}=\mathscr{G}_{0} \oplus \mathscr{G}_{1} \oplus \cdots \oplus \mathscr{G}_{m}$ and $\mathscr{G}^{\prime}=\mathscr{G}_{0}^{\prime} \oplus \mathscr{G}_{1}^{\prime} \oplus \cdots \oplus \mathscr{G}_{n}^{\prime}$ be two reducible Carnot algebras written as direct sums of an abelian factor and irreducible nonabelian factors. Let $\phi: \mathscr{G} \rightarrow \mathscr{G}^{\prime}$ be a graded isomorphism. Then $\phi\left(\mathscr{G}_{0}\right)=\mathscr{G}_{0}^{\prime}$.

Proof. By Lemma 2.3, $\phi\left(\mathscr{G}_{0}\right) \subset \mathscr{G}_{0}^{\prime}$. Since $\phi$ is an isomorphism, the conclusion follows by considering $\phi^{-1}$.

Proposition 2.5 treats the case when the abelian factor is trivial.

Proposition 2.5. Let $\mathscr{G}=\mathscr{G}_{0} \oplus \mathscr{G}_{1} \oplus \cdots \oplus \mathscr{G}_{m}$ and $\mathscr{G}^{\prime}=\mathscr{G}_{0}^{\prime} \oplus \mathscr{G}_{1}^{\prime} \oplus \cdots \oplus \mathscr{G}_{n}^{\prime}$ be two reducible Carnot algebras written as direct sums of an abelian factor and irreducible nonabelian factors. Let $\phi: \varphi \rightarrow \varphi^{\prime}$ be a graded isomorphism. Suppose $\mathscr{G}$ has no abelian factor (that is, $\mathscr{G}_{0}=\{0\}$ ). Then $\mathscr{G}_{0}^{\prime}=\{0\}, m=n$ and after possibly permuting the factors $\mathscr{G}_{1}^{\prime}, \ldots, \mathscr{G}_{m}^{\prime}$, there exist graded isomorphisms $\phi_{i}: \mathscr{G}_{i} \rightarrow \mathscr{G}_{i}^{\prime}$ such that $\phi=\phi_{1} \oplus \cdots \oplus \phi_{m}$.

Now we start the proof of Proposition 2.5. First observe that Proposition 2.4 implies $\mathscr{G}_{0}^{\prime}=\{0\}$. In the following proofs, we shall use both decompositions $\mathscr{G}=V_{1} \oplus \cdots \oplus V_{r}=\mathscr{G}_{1} \oplus \cdots \oplus \mathscr{G}_{m}$ of $\mathscr{G}$, as well as those for $\mathscr{G}^{\prime}$. 
Lemma 2.6. Let $x \in V_{1}$. Write $x=x_{i_{1}}+\cdots+x_{i_{k}}\left(1 \leq i_{1}<\cdots<i_{k} \leq m\right)$ with $x_{i_{j}} \in\left(\mathscr{G}_{i_{j}} \cap V_{1}\right) \backslash\{0\}$. If $k \geq 2$, then $d\left(x_{i_{j}}\right)>d(x)$.

Proof. We first show that $\operatorname{ker}(\operatorname{ad} x) \subset \operatorname{ker}\left(\operatorname{ad} x_{i_{j}}\right)$ for all $1 \leq j \leq k$. Let $y \in \operatorname{ker}(\operatorname{ad} x)$. Write $y=y_{1}+\cdots+y_{m}$ with $y_{i} \in \mathscr{G}_{i}$. Then $0=[x, y]=\left[x_{i_{1}}, y_{i_{1}}\right]+\cdots+\left[x_{i_{k}}, y_{i_{k}}\right]$. Since $\left[x_{i_{j}}, y_{i_{j}}\right] \in \mathscr{G}_{i_{j}}$, we have $\left[x_{i_{j}}, y_{i_{j}}\right]=0$. Hence $\left[x_{i_{j}}, y\right]=\left[x_{i_{j}}, y_{i_{j}}\right]=0$; that is, $y \in \operatorname{ker}\left(\operatorname{ad} x_{i_{j}}\right)$.

Next we shall find an element $y \in \operatorname{ker}\left(\operatorname{ad} x_{i_{j}}\right) \backslash \operatorname{ker}(\operatorname{ad} x)$. Since $k \geq 2$, there is some $1 \leq l \leq k$ with $l \neq j$. By Lemma 2.2, since $\varphi_{i_{l}}$ is nonabelian and irreducible, there is some $y \in \mathscr{G}_{i_{l}}$ such that $\left[x_{i_{l}}, y\right] \neq 0$. Now notice that $\left[x_{i_{j}}, y\right]=0$ and $[x, y]=\left[x_{i_{l}}, y\right] \neq 0$.

For each $1 \leq i \leq m$, set

$$
A_{i}=\left\{x \in \mathscr{G}_{i} \cap V_{1}: \phi(x) \in \mathscr{G}_{j}^{\prime} \text { for some } j\right\} .
$$

Let $N_{i} \subset \mathscr{G}_{i} \cap V_{1}$ be the vector subspace spanned by $A_{i}$. Similarly, for each $1 \leq j \leq n$ set

$$
A_{j}^{\prime}=\left\{y \in \mathscr{G}_{j}^{\prime} \cap V_{1}^{\prime}: \phi^{-1}(y) \in \mathscr{G}_{i} \text { for some } i\right\} .
$$

Let $N_{j}^{\prime} \subset \mathscr{G}_{j}^{\prime} \cap V_{1}^{\prime}$ be the vector subspace spanned by $A_{j}^{\prime}$.

Lemma 2.7. We have $N_{i}=\mathscr{G}_{i} \cap V_{1}$ for each $i$ and $N_{j}^{\prime}=\mathscr{G}_{j}^{\prime} \cap V_{1}^{\prime}$ for each $j$.

Proof. We prove by contradiction. Suppose $N_{i} \neq \mathscr{G}_{i} \cap V_{1}$ for some $i$ or $N_{j}^{\prime} \neq \mathscr{G}_{j}^{\prime} \cap V_{1}^{\prime}$ for some $j$. Let $d_{1}=0$ if $N_{i}=\mathscr{G}_{i} \cap V_{1}$ for all $i$; otherwise, let

$$
d_{1}=\max \left\{d(x): x \in\left(\varphi_{i} \cap V_{1}\right) \backslash N_{i} \text { for some } i\right\} .
$$

Similarly, let $d_{2}=0$ if $N_{j}^{\prime}=\varphi_{j}^{\prime} \cap V_{1}^{\prime}$ for all $j$; otherwise, let

$$
d_{2}=\max \left\{d(y): y \in\left(\mathscr{G}_{j}^{\prime} \cap V_{1}^{\prime}\right) \backslash N_{j}^{\prime} \text { for some } j\right\} .
$$

Let $d_{0}=\max \left\{d_{1}, d_{2}\right\}$. After possibly replacing $\phi$ with $\phi^{-1}$, we may assume $d_{0}=d_{1}$. Pick $x \in\left(G_{i} \cap V_{1}\right) \backslash N_{i}$ (for some $i$ ) with $d(x)=d_{0}$. By the definition of $N_{i}$ we have $x \notin A_{i}$. Hence $\phi(x)$ can be written as

$$
\phi(x)=y_{1}+\cdots+y_{k},
$$

where $k \geq 2$ and $y_{s} \in\left(\varphi_{j_{s}^{\prime}}^{\prime} \cap V_{1}^{\prime}\right) \backslash\{0\}$ for each $1 \leq s \leq k$, and $1 \leq j_{1}<\cdots<j_{k} \leq n$. By Lemma 2.6, $d\left(y_{s}\right)>d(\phi(x))=d(x)=d_{0}$. It follows from the definition of $d_{0}$ that $y_{s} \in N_{j_{s}}^{\prime}$. Hence there is an expression

$$
y_{s}=z_{s, 1}+\cdots+z_{s, u_{s}}+w_{s, 1}+\cdots+w_{s, v_{s}}
$$


with $z_{s, p}, w_{s, q} \in A_{j_{s}}^{\prime}$ such that $\phi^{-1}\left(z_{s, p}\right) \in \mathscr{G}_{i} \cap V_{1}$ and $\phi^{-1}\left(w_{s, q}\right) \in \mathscr{G}_{t} \cap V_{1}$ for some $t \neq i$ (here $t$ may depend on $q$ ). Notice that (2-2) and (2-3) imply

$$
x=\sum_{s, p} \phi^{-1}\left(z_{s, p}\right)+\sum_{s, q} \phi^{-1}\left(w_{s, q}\right) .
$$

Since $x \in \mathscr{G}_{i}$ and $\mathscr{G}_{=}=\mathscr{G}_{1} \oplus \cdots \oplus \mathscr{G}_{m}$ is a direct sum decomposition, we obtain $x=\sum_{s, p} \phi^{-1}\left(z_{s, p}\right)$. Notice that each $\phi^{-1}\left(z_{s, p}\right) \in A_{i}$. It follows that $x \in N_{i}$, contradicting the assumption.

Lemma 2.8. For each $i$, there is some $j$ such that $\phi\left(\mathscr{G}_{i} \cap V_{1}\right) \subset \mathscr{G}_{j}^{\prime} \cap V_{1}^{\prime}$.

Proof. Fix $i$. By Lemma 2.7, $\varphi_{i} \cap V_{1}=N_{i}$. Hence there is a vector space basis $B$ of $G_{i} \cap V_{1}$ consisting of elements of $A_{i}$. Write $B$ as a disjoint union $B=\sqcup B_{j}$, where $B_{j}$ consists of those elements of $B$ that are mapped into $\varphi_{j}^{\prime}$ under $\phi$. Since $\phi$ is an isomorphism and $\mathscr{G}_{j_{1}}$ and $\mathscr{G}_{j_{2}}^{\prime}$ commute for $j_{1} \neq j_{2}$, we see that $[X, Y]=0$ for $X \in B_{j_{1}}$ and $Y \in B_{j_{2}}$. Let $E_{j} \subset \varphi_{i}$ be the subalgebra of $\varphi_{i}$ generated by $B_{j}$. Observe that $E_{j}$ is an ideal of $\varphi_{i}$ and $\varphi_{i}$ admits the direct sum decomposition $\varphi_{i}=E_{1} \oplus \cdots \oplus E_{n}$. Since $\varphi_{i}$ is irreducible, $E_{j}=\{0\}$ for all $j$ except exactly one. It follows that for some $j$, all the elements in $B$ are mapped into $\mathscr{G}_{j}^{\prime}$. Since $B$ is a basis of $\varphi_{i} \cap V_{1}$, we have $\phi\left(\varphi_{i} \cap V_{1}\right) \subset \mathscr{G}_{j}^{\prime}$.

Applying Lemma 2.8 to $\phi^{-1}$, we see that for each $j$, there is some $i$ such that $\phi^{-1}\left(\mathscr{G}_{j}^{\prime} \cap V_{1}^{\prime}\right) \subset \mathscr{G}_{i} \cap V_{1}$. From this it is easy to see that $m=n$, and after possibly permuting the factors $\mathscr{G}_{j}^{\prime}$ we have $\phi\left(\mathscr{G}_{i}\right)=\mathscr{G}_{i}^{\prime}$. Proposition 2.5 follows.

\section{Quasisymmetric homeomorphisms are bilipschitz}

In this section we show that in most cases quasisymmetric homeomorphisms between reducible Carnot groups are bilipschitz.

A simply connected nilpotent Lie group is a Carnot group if its Lie algebra is a Carnot algebra. Let $G$ be a Carnot group with Lie algebra $G=V_{1} \oplus \cdots \oplus V_{r}$. The subspace $V_{1}$ defines a left invariant distribution $H G \subset T G$ on $G$. We fix a left invariant inner product on $H G$. An absolutely continuous curve $\gamma$ in $G$ whose velocity vector $\gamma^{\prime}(t)$ is contained in $H_{\gamma(t)} G$ for a.e. $t$ is called a horizontal curve. By Chow's theorem ([Bellaïche and Risler 1996], Theorem 2.4), any two points of $G$ can be connected by horizontal curves. Let $p, q \in G$; the Carnot distance $d(p, q)$ between them is defined as the infimum of length of horizontal curves that join $p$ and $q$.

Since the inner product on $H G$ is left invariant, the Carnot metric on $G$ is also left invariant. Different choices of inner product on $H G$ result in Carnot metrics that are bilipschitz equivalent. The Hausdorff dimension of $G$ with respect to a Carnot metric is given by $\sum_{i=1}^{r} i \cdot \operatorname{dim} V_{i}$. We use the corresponding Hausdorff measure on $G$. When $G=G_{1} \times G_{2}$ is a direct product of two Carnot groups (with 
a suitable choice of inner product on $H G$ ), the Carnot metric on $G$ is the product of the Carnot metrics on $G_{1}$ and $G_{2}$, and the Hausdorff measure on $G$ is the product of the Hausdorff measures on $G_{1}$ and $G_{2}$.

Recall that, for a simply connected nilpotent Lie group $G$ with Lie algebra $\mathscr{G}$, the exponential map exp $: \mathscr{G} \rightarrow G$ is a diffeomorphism. Furthermore, the exponential map induces a one-to-one correspondence between Lie subalgebras of $\mathscr{G}$ and connected Lie subgroups of $G$.

Let $G$ be a Carnot group with Lie algebra $\mathscr{G}=V_{1} \oplus \cdots \oplus V_{r}$. Since $\lambda_{t}: \mathscr{G} \rightarrow \mathscr{G}$ $(t>0)$ is a Lie algebra automorphism and $G$ is simply connected, there is a unique Lie group automorphism $\Lambda_{t}: G \rightarrow G$ whose differential at the identity is $\lambda_{t}$. For each $t>0, \Lambda_{t}$ is a similarity with respect to the Carnot metric: $d\left(\Lambda_{t}(p), \Lambda_{t}(q)\right)=$ $t d(p, q)$ for any two points $p, q \in G$. A Lie group homomorphism $f: G \rightarrow G^{\prime}$ between two Carnot groups is a graded homomorphism if it commutes with $\Lambda_{t}$ for all $t>0$; that is, if $f \circ \Lambda_{t}=\Lambda_{t} \circ f$. Notice that a Lie group homomorphism $f: G \rightarrow G^{\prime}$ between two Carnot groups is graded if and only if the corresponding Lie algebra homomorphism is graded.

A Carnot group is reducible if its Lie algebra is reducible. Equivalently, a Carnot group is reducible if it is isomorphic to the direct product of two Carnot groups. A Carnot group is called irreducible otherwise.

Proposition 2.4 and Proposition 2.5 respectively immediately imply Corollary 3.1 and Corollary 3.2, which say that a graded isomorphism of reducible Carnot groups preserves certain Lie subgroups.

Corollary 3.1. Let $G=G_{0} \times G_{1} \times \cdots \times G_{m}$ and $G^{\prime}=G_{0}^{\prime} \times G_{1}^{\prime} \times \cdots \times G_{n}^{\prime}$ be two reducible Carnot groups written as direct products of an abelian factor and irreducible nonabelian factors. Let $f: G \rightarrow G^{\prime}$ be a graded isomorphism. Then $f\left(G_{0}\right)=G_{0}^{\prime}$.

Corollary 3.2. Let $G=G_{0} \times G_{1} \times \cdots \times G_{m}$ and $G^{\prime}=G_{0}^{\prime} \times G_{1}^{\prime} \times \cdots \times G_{n}^{\prime}$ be two reducible Carnot groups written as direct products of an abelian factor and irreducible nonabelian factors. Let $f: G \rightarrow G^{\prime}$ be a graded isomorphism. Suppose $G$ has no abelian factor (that is, $G_{0}=\{e\}$ ). Then $G_{0}^{\prime}=\{e\}, m=n$ and after possibly permuting the factors $G_{1}^{\prime}, \ldots, G_{m}^{\prime}$, there exist graded isomorphisms $f_{i}: G_{i} \rightarrow G_{i}^{\prime}$ such that $f=f_{1} \times \cdots \times f_{m}$.

Definition 3-1. Let $G$ and $G^{\prime}$ be two Carnot groups endowed with Carnot metrics. A map $F: G \rightarrow G^{\prime}$ is Pansu-differentiable at $x \in G$ if there exists a graded homomorphism $L: G \rightarrow G^{\prime}$ such that

$$
\lim _{y \rightarrow x} \frac{d\left(F(x)^{-1} F(y), L\left(x^{-1} y\right)\right)}{d(x, y)}=0 .
$$


In this case, the graded homomorphism $L: G \rightarrow G^{\prime}$ is called the Pansu differential of $F$ at $x$, and is denoted by $d F(x)$.

Definition 3-2. Let $\eta:[0, \infty) \rightarrow[0, \infty)$ be a homeomorphism. A homeomorphism of metric spaces $F: X \rightarrow Y$ is an $\eta$-quasisymmetric homeomorphism if for all distinct triples $x, y, z \in X$, we have

$$
\frac{d(F(x), F(y))}{d(F(x), F(z))} \leq \eta\left(\frac{d(x, y)}{d(x, z)}\right) .
$$

A map $F: X \rightarrow Y$ is a quasisymmetric homeomorphism if it is an $\eta$-quasisymmetric homeomorphism for some $\eta$.

The following result (except the terminology) is due to Pansu [1989].

Theorem 3.3. Let $F: G \rightarrow G^{\prime}$ be a quasisymmetric homeomorphism between two Carnot groups. Then $F$ is a.e. Pansu-differentiable. Furthermore, at a.e. $x \in G$, the Pansu differential $d F(x): G \rightarrow G^{\prime}$ is a graded isomorphism.

In Theorem 3.3 and the proofs below, "a.e." is with respect to the Hausdorff measure on $G$.

For the proof of Theorem 1.1, we need the following:

Proposition 3.4. Let $G$ and $G^{\prime}$ be two Carnot groups, $W \subset V_{1}, W^{\prime} \subset V_{1}^{\prime}$ be subspaces. Denote by $\mathscr{G}_{W} \subset \mathscr{G}_{\text {and }} \mathscr{G}_{W^{\prime}}^{\prime} \subset \mathscr{G}^{\prime}$, respectively, the Lie subalgebras generated by $W$ and $W^{\prime}$. Let $H \subset G$ and $H^{\prime} \subset G^{\prime}$, respectively, be the connected Lie subgroups of $G$ and $G^{\prime}$ corresponding to $\mathscr{G}_{W}$ and $\mathscr{G}_{W^{\prime}}^{\prime}$. Let $F: G \rightarrow G^{\prime}$ be a quasisymmetric homeomorphism. If $d F(x)(W) \subset W^{\prime}$ for a.e. $x \in G$, then $F$ sends left cosets of $H$ into left cosets of $H^{\prime}$.

Proof. For each nonzero vector $u \in W$, the $\operatorname{set}\{\exp (t u): t \in \mathbb{R}\}$ is a subgroup of $G$. It is a geodesic with respect to the Carnot metric and shall be called a horizontal line. For each nonzero vector $u \in W$, let $\mathscr{F}_{u}$ be the set of left cosets of $\{\exp (t u): t \in \mathbb{R}\}$ in $G$. By the main result in [Balogh et al. 2007], $F: G \rightarrow G^{\prime}$ is absolutely continuous on almost every curve. It follows that for almost every $L \in \mathscr{F}_{u}$, the map $\left.F\right|_{L}: L \rightarrow G^{\prime}$ is an absolutely continuous curve in $G^{\prime}$. On the other hand, by Pansu's theorem, $F$ is a.e. Pansu-differentiable and the Pansu differential $d F(x): G \rightarrow G^{\prime}$ is a graded isomorphism for a.e. $x \in G$. Also by assumption, $d F(x)(W) \subset W^{\prime}$ for a.e. $x \in G$. It follows from Fubini's theorem that, for almost every $L \in \mathscr{F}_{u}$, the Pansu differential $d F(x): G \rightarrow G^{\prime}$ exists, is a graded isomorphism and satisfies $d F(x)(W) \subset W^{\prime}$ for a.e. $x \in L$. Hence, the tangent vectors of the curve $\left.F\right|_{L}$ lie in $W^{\prime}$ almost everywhere. It follows that for almost every $L \in \mathscr{F}_{u}, F(L)$ lies in a left coset of $H^{\prime}$. Now the continuity of $F$ and a limiting argument show that the same is true for all $L \in \mathscr{F}_{u}$. Conceivably, it might be possible for distinct $L_{1}, L_{2} \in \mathscr{F}_{u}$ to lie in the same left 
coset of $H$, while their images $F\left(L_{1}\right)$ and $F\left(L_{2}\right)$ lie in distinct cosets of $H^{\prime}$. We next show that this cannot happen.

In a Carnot group, every two points can be joined by a piecewise geodesic, where each piece is a left translation of a segment in a horizontal line. The preceding paragraph shows that the image under $F$ of each piece lies in a left coset of $H^{\prime}$. It follows that the image of the entire piecewise geodesic lies in a left coset of $H^{\prime}$. Hence $F$ sends left cosets of $H$ into left cosets of $H^{\prime}$.

Now we are ready to prove Theorem 1.1 .

Proof of Theorem 1.1. Let $F: G \rightarrow G^{\prime}$ be a quasisymmetric homeomorphism between two Carnot groups. Suppose $G$ is reducible and admits a direct product decomposition of irreducible Carnot groups where at least two of the factors are not isomorphic. We first use Proposition 3.4 to show that $F$ preserves a certain foliation. The arguments in [Shanmugalingam and Xie 2012] then show that $F$ is bilipschitz.

Write $G=G_{0} \times G_{1} \times \cdots \times G_{m}$ and $G^{\prime}=G_{0}^{\prime} \times G_{1}^{\prime} \times \cdots \times G_{n}^{\prime}$, where $G_{0}, G_{0}^{\prime}$ are abelian and $G_{i}, G_{j}^{\prime}$ are irreducible nonabelian factors.

First consider the case when $G_{0}$ is nontrivial. Let $\mathscr{F}$ be the foliation of $G$ consisting of the cosets of $G_{0}$, and similarly let $\mathscr{F}^{\prime}$ be the foliation of $G^{\prime}$ consisting of the cosets of $G_{0}^{\prime}$. The leaf space of $\mathscr{F}$ can be naturally identified with $N:=G_{1} \times \cdots \times G_{m}$, and that of $\mathscr{F}^{\prime}$ with $N^{\prime}:=G_{1}^{\prime} \times \cdots \times G_{n}^{\prime}$. By Corollary 3.1 and Proposition 3.4, the map $F$ sends the leafs of $\mathscr{F}_{F}$ to the leafs of $\mathscr{F}^{\prime}$. Hence $F$ induces a map $F_{1}: N \rightarrow N^{\prime}$. Notice that $G=G_{0} \times N$ with the Carnot metric is isometric to the product of $G_{0}$ and $N$ (also equipped with the Carnot metric); similarly for $G^{\prime}$. The arguments in [Shanmugalingam and Xie 2012] go through and imply that $F_{1}$ is also quasisymmetric. Since both the leafs and the leaf spaces are geodesic metric spaces, the arguments further show that $F$ is bilipschitz.

Next we consider the case when $G_{0}$ is trivial. Then $G=G_{1} \times \cdots \times G_{m}$ is a direct product of nonabelian irreducible Carnot groups. We combine all isomorphic factors in the above decomposition to obtain $G=N_{1} \times \cdots \times N_{s}$. Each $N_{j}$ is a direct product of isomorphic nonabelian irreducible Carnot groups, and the factors in $N_{i}$ and $N_{j}$ are not isomorphic for $i \neq j$. Similarly, $G^{\prime}$ can also be written as such a product $G^{\prime}=N_{1}^{\prime} \times \cdots \times N_{t}^{\prime}$. Notice that the assumption of Theorem 1.1 implies that $s \geq 2$. Corollary 3.2 implies that $s=t$, and after possibly permuting the factors $N_{i}^{\prime}$, the Pansu differential satisfies $d F(x)\left(N_{i}\right)=N_{i}^{\prime}$ for all $i$ and a.e. $x \in N$. Now the arguments in the preceding paragraph show that $F$ is bilipschitz. The proof of Theorem 1.1 is now complete.

\section{Acknowledgments}

I thank Bruce Kleiner for helpful discussions, and the referee for useful remarks. 


\section{References}

[Balogh et al. 2007] Z. M. Balogh, P. Koskela, and S. Rogovin, "Absolute continuity of quasiconformal mappings on curves", Geom. Funct. Anal. 17:3 (2007), 645-664. MR 2009g:30023 Zbl 1134.30014

[Bellaïche and Risler 1996] A. Bellaïche and J.-J. Risler (editors), Sub-Riemannian geometry (Paris, 1992), Progress in Mathematics 144, Birkhäuser, Basel, 1996. MR 97f:53002 Zbl 0848.00020

[Bonk and Schramm 2000] M. Bonk and O. Schramm, "Embeddings of Gromov hyperbolic spaces", Geom. Funct. Anal. 10:2 (2000), 266-306. MR 2001g:53077 Zbl 0972.53021

[Heinonen and Koskela 1998] J. Heinonen and P. Koskela, "Quasiconformal maps in metric spaces with controlled geometry”, Acta Math. 181:1 (1998), 1-61. MR 99j:30025 Zbl 0915.30018

[Heintze 1974] E. Heintze, “On homogeneous manifolds of negative curvature”, Math. Ann. 211 (1974), 23-34. MR 50 \#5695 Zbl 0273.53042

[Pansu 1989] P. Pansu, "Métriques de Carnot-Carathéodory et quasiisométries des espaces symétriques de rang un", Ann. of Math. (2) 129:1 (1989), 1-60. MR 90e:53058 Zbl 0678.53042

[Shanmugalingam and Xie 2012] N. Shanmugalingam and X. Xie, "A rigidity property of some negatively curved solvable Lie groups", Comment. Math. Helv. 87:4 (2012), 805-823. MR 2984572 Zbl 1255.22005

Received May 17, 2012. Revised May 5, 2013.

Xiangdong Xie

DEPARTMENT OF MATHEMATICS AND STATistics

BOWLING GREEN STATE UNIVERSITY

BOWLING GREEN, OH 43403

UNITED STATES

xiex@bgsu.edu 


\title{
PACIFIC JOURNAL OF MATHEMATICS
}

\author{
msp.org/pjm
}

Founded in 1951 by E. F. Beckenbach (1906-1982) and F. Wolf (1904-1989)

\section{EDITORS}

V. S. Varadarajan (Managing Editor)

Department of Mathematics

University of California

Los Angeles, CA 90095-1555

pacific@math.ucla.edu

Paul Balmer

Department of Mathematics

University of California

Los Angeles, CA 90095-1555

balmer@math.ucla.edu

Daryl Cooper

Department of Mathematics

University of California

Santa Barbara, CA 93106-3080 cooper@math.ucsb.edu

Jiang-Hua $\mathrm{Lu}$

Department of Mathematics

Pokfulam Rd., Hong Kong jhlu@maths.hku.hk
The University of Hong Kong

Don Blasius

Department of Mathematics University of California

Los Angeles, CA 90095-1555

blasius@math.ucla.edu

Robert Finn

Department of Mathematics Stanford University

Stanford, CA 94305-2125

finn@math.stanford.edu

Sorin Popa

Department of Mathematics

University of California

Los Angeles, CA 90095-1555 popa@math.ucla.edu

Paul Yang

Department of Mathematics Princeton University

Princeton NJ 08544-1000

yang@math.princeton.edu

\section{PRODUCTION}

Silvio Levy, Scientific Editor, production@msp.org

\section{SUPPORTING INSTITUTIONS}

ACADEMIA SINICA, TAIPEI

CALIFORNIA INST. OF TECHNOLOGY

INST. DE MATEMÁTICA PURA E APLICADA

KEIO UNIVERSITY

MATH. SCIENCES RESEARCH INSTITUTE

NEW MEXICO STATE UNIV.

OREGON STATE UNIV.

\author{
STANFORD UNIVERSITY \\ UNIV. OF BRITISH COLUMBIA \\ UNIV. OF CALIFORNIA, BERKELEY \\ UNIV. OF CALIFORNIA, DAVIS \\ UNIV. OF CALIFORNIA, LOS ANGELES \\ UNIV. OF CALIFORNIA, RIVERSIDE \\ UNIV. OF CALIFORNIA, SAN DIEGO \\ UNIV. OF CALIF., SANTA BARBARA
}

\author{
Vyjayanthi Chari \\ Department of Mathematics \\ University of California \\ Riverside, CA 92521-0135 \\ chari@math.ucr.edu \\ Kefeng Liu \\ Department of Mathematics \\ University of California \\ Los Angeles, CA 90095-1555 \\ liu@math.ucla.edu \\ Jie Qing \\ Department of Mathematics \\ University of California \\ Santa Cruz, CA 95064 \\ qing@cats.ucsc.edu
}

These supporting institutions contribute to the cost of publication of this Journal, but they are not owners or publishers and have no responsibility for its contents or policies.

See inside back cover or msp.org/pjm for submission instructions.

The subscription price for 2013 is US \$400/year for the electronic version, and \$485/year for print and electronic.

Subscriptions, requests for back issues and changes of subscribers address should be sent to Pacific Journal of Mathematics, P.O. Box 4163, Berkeley, CA 94704-0163, U.S.A. The Pacific Journal of Mathematics is indexed by Mathematical Reviews, Zentralblatt MATH, PASCAL CNRS Index, Referativnyi Zhurnal, Current Mathematical Publications and the Science Citation Index.

The Pacific Journal of Mathematics (ISSN 0030-8730) at the University of California, c/o Department of Mathematics, 798 Evans Hall \#3840, Berkeley, CA 94720-3840, is published twelve times a year. Periodical rate postage paid at Berkeley, CA 94704, and additional mailing offices. POSTMASTER: send address changes to Pacific Journal of Mathematics, P.O. Box 4163, Berkeley, CA 94704-0163.

PJM peer review and production are managed by EditFLOW ${ }^{\circledR}$ from Mathematical Sciences Publishers.

\section{PUBLISHED BY}

mathematical sciences publishers

nonprofit scientific publishing

http://msp.org/

(C) 2013 Mathematical Sciences Publishers 


\section{PACIFIC JOURNAL OF MATHEMATICS}

Volume $265 \quad$ No. $1 \quad$ September 2013

Genus-two Goeritz groups of lens spaces

SANGBUM CHO

A compact embedding theorem for generalized Sobolev spaces

SENG-KeE ChUa, SCOTT Rodney and Richard L. WheEden

Partial integrability of almost complex structures and the existence of

solutions for quasilinear Cauchy-Riemann equations

CHONG-KYU HAN and JONG-DO PARK

An overdetermined problem in potential theory

DMITRY KHAVINSON, ERIK LUNDBERG and RAZVAN TEODORESCU

Quasisymmetric homeomorphisms on reducible Carnot groups

XIANGDONG XIE

Capillarity and Archimedes' principle

JOHN MCCUAN and RAY TREINEN

Generalized eigenvalue problems of nonhomogeneous elliptic operators and their application

DUmitru Motreanu and Mieko TANaKa

Weighted Ricci curvature estimates for Hilbert and Funk geometries

SHIN-ICHI OHTA

On generalized weighted Hilbert matrices

EMMANUEL PREISSMANN and OLIVIER LÉVÊQUE

Unique prime decomposition results for factors coming from wreath product 221 groups

J. OWEN SizEMORE and ADAM WinCHESTER

On volume growth of gradient steady Ricci solitons

Guofang Wei and Peng Wu

Classification of moduli spaces of arrangements of nine projective lines

FEI YE 